\title{
Simultaneous Bilateral Pneumonectomy for Early Multiple Primary Lung Cancer Feasibility Analysis
}

\author{
Zhonglong Zheng* , Tao Li, Yang Chen, Yang Zhang, Pan Zhang \\ Department of Thoracic Surgery, Ziyang People's Hospital, Ziyang 641300, Sichuan Province, China
}

\begin{abstract}
[Abstract] Objective: To analyze the feasibility of simultaneous bilateral thoracoscopic lung resection in the treatment of multiple primary lung cancers in the early stage. Methods: The study time range is between March 2019 and March 2021. A sample of 30 patients with early multiple primary lung cancer admitted to this hospital were included, and they were divided into a study group, a control group, and samples within the group using a random number table scheme $\mathrm{n}=15$, patients in the control group underwent staged bilateral thoracoscopic pneumonectomy, and patients in the study group underwent bilateral thoracoscopic pneumonectomy at the same time. The indicators of the two groups were compared and analyzed. Results: There was no significant difference in the operation time and intraoperative blood loss between the two groups ( $>0.05)$. There were significant differences in the VAS score, total length of hospital stay, and total surgical costs on the first day after surgery $(\mathrm{P}<0.05)$; there was no significant difference in the two groups' postoperative recovery indicators and the incidence of complications $(\mathrm{P}>0.05)$. Conclusion: It is safe and feasible to treat patients with multiple primary lung cancer in both lungs at the same time with simultaneous bilateral thoracoscopic surgery, and is suitable for promotion.
\end{abstract}

Key words: The same period; Bilateral thoracoscopic lung resection; Early multiple primary lung cancer

Publication date: May, 2021; Publication online: 31 May, 2021

*Corresponding author: Zhonglong Zheng, 346925260@qq.com

\section{Introduction}

Lung cancer, also known as primary bronchial lung cancer, is a malignant tumor that originates from the lungs, bronchus, trachea, and other areas. Specific pathological types include large cell carcinoma, small cell carcinoma, squamous cell carcinoma, adenocarcinoma, etc. Patients with lung cancer have no typical clinical manifestations at the initial stage of onset, and can induce sputum expectoration, hemoptysis, chest pain, and dyspnea as the condition changes. The mortality rate is high, which has a serious impact on the quality of life of patients ${ }^{[1]}$. Early multiple primary lung cancers belong to the category of non-small cell lung cancer, which can occur in bilateral or unilateral lung lobes, including multiple histological types. The main programs for clinical diagnosis of early multiple primary lung cancer are bilateral thoracoscopic lobectomy, sublobectomy combined with lymph node dissection, and ipsilateral bilateral thoracoscopic lobectomy combined with lymph node dissection, etc ${ }^{[2]}$. There are still controversies about the clinical efficacy of concurrent or staged surgical treatment. This study summarizes and evaluates the basic data of early multiple primary lung cancer patients in our hospital, and studies and evaluates the related issues of bilateral thoracoscopic lung resection during the same period.

\section{Materials and methods}

\subsection{General information}

The study time range is from March 2019 to March 2021. A sample of 30 patients with early multiple primary lung cancer were admitted to this hospital, and they were divided into a study group and a control group using a random number table scheme. The samples within the group were $n=$ 15. The basic data of the two groups of patients were 
summarized and analyzed. The study group had 8 males and 7 females. The age range spanned from 40 to 58 years old, with an average of (49.58 \pm 2.44$)$ years old, and the tumor diameter is $(2.54 \pm 1.07) \mathrm{cm}$. In the control group, there were 9 males and 6 females. The age ranges from 41 to 60 years old, with an average of $(50.27 \pm 2.47)$ years old, and tumor diameter $(2.59 \pm 1.04) \mathrm{cm}$. Differences in baseline data had no effect on the conclusion of this study $(\mathrm{P}>0.05)$.

Inclusion criteria: Early-stage multiple primary lung cancer was diagnosed by $\mathrm{CT}$ and pathological examination, no distant metastasis or lymph node metastasis, maximum tumor diameter was less than $3.0 \mathrm{~cm}$, in line with surgical indications, and agreed to participate in this study.

Exclusion criteria: Patients with other malignant tumors, no indications for surgery, and other patients who cannot cooperate with this study.

\subsection{Methods}

Before surgery, both groups of patients underwent basic examinations such as cranial MRI, enhanced CT of the chest and above, and abdominal ultrasound. Patients in the study group underwent bilateral thoracoscopic pneumonectomy at the same time. The physician evaluated the size, density, and location of the patient's lesions, and selected appropriate surgical treatment options based on the results of frozen histopathological examinations and the status of the remaining lung nodules, including unilateral lobectomy + Unilateral segmental or wedge resection (sublobectomy); bilateral segmental resection or wedge resection. Single-port or three-port thoracoscopy was used for lobe and segment resection, and single-port thoracoscopy was used for unilateral wedge resection. Sampling or lymph node dissection was performed after the resection was completed.
The patients in the control group underwent staged bilateral thoracoscopic pneumonectomy. The operation plan was the same as that in the study group, and the operation interval was 3-6 months. After the operation, the two groups of patients were followed up and regularly reviewed by taking tumor markers, chest CT, abdominal ultrasound, MRI, whole body bone scan, etc. The treatment plan was adjusted according to the results of the review.

Evaluation criteria

Intraoperative blood loss, operation time, VAS score on the first day after operation, total hospital stay, total operation cost and other indicators were compared between the two groups. Statistics on the related indicators of postoperative recovery and the incidence of various complications of the two groups of patients.

Statistical methods

SPSS23.0 software was used to calculate various data. In this study, the measurement data is $\left(x_{ \pm s}\right)$, the test method is $t$, the count data is (\%), and the test method is $\chi 2$. If $\mathrm{P}<0.05$, there is a difference between the groups.

\section{Results}

3.1 Comparison of the intraoperative blood loss, operation time and VAS score on the first day after surgery, total hospital stay and cost indicators

There was no significant difference in operation time and intraoperative blood loss between the two groups $(\mathrm{P}>0.05)$; the VAS score on the first day after surgery was higher in the study group than in the control group $(\mathrm{P}<0.05)$; the total length of hospital stay and total surgical expenses in the study group were significantly lower than the control group $(\mathrm{P}<0.05)$ (Table 1).

Table 1. Comparison of intraoperative blood loss, operation time and VAS score on the first day after operation, total hospital stay and cost indicators $\left(x_{ \pm s}\right)$

\begin{tabular}{cccccc}
\hline Group & $\begin{array}{c}\text { Intraoperative } \\
\text { blood loss }(\mathbf{m l})\end{array}$ & $\begin{array}{c}\text { Operating time } \\
(\mathbf{m i n})\end{array}$ & $\begin{array}{c}\text { VAS score on the } \\
\text { first day after } \\
\text { operation }\end{array}$ & $\begin{array}{c}\text { Total hospital } \\
\text { stay (d) }\end{array}$ & $\begin{array}{c}\text { Total hospital } \\
\text { cost (yuan) }\end{array}$ \\
\hline $\begin{array}{c}\text { Study group } \\
(\mathrm{n}=15)\end{array}$ & $94.42 \pm 7.15$ & $185.77 \pm 48.35$ & $2.79 \pm 0.48$ & $7.25 \pm 1.69$ & $22018.62 \pm 827.79$ \\
$\begin{array}{c}\text { Control group } \\
(\mathrm{n}=15)\end{array}$ & $95.26 \pm 5.43$ & $185.68 \pm 22.79$ & $2.03 \pm 0.27$ & $13.86 \pm 2.77$ & $30577.79 \pm 869.92$ \\
$\mathrm{t}$ & 0.362 & 0.006 & 5.344 & 7.889 & 27.605 \\
$\mathrm{P}$ & 0.719 & 0.994 & 0.000 & 0.000 & 0.000 \\
\hline
\end{tabular}

\subsection{Comparison of related indicators of postoperative recovery between the two groups}


There was no significant difference between the two groups

2).

of related indexes of postoperative recovery $(\mathrm{P}>0.05)$ (Table

Table 2. Comparison of related indexes of postoperative recovery between the two groups $\left(\bar{x}_{ \pm \mathrm{s}}, \mathrm{d}\right)$

\begin{tabular}{ccc}
\hline Group & Postoperative activity time & Extubation time \\
\hline Study group $(\mathrm{n}=15)$ & $2.84 \pm 0.22$ & $3.03 \pm 0.49$ \\
Control group $(\mathrm{n}=15)$ & $2.91 \pm 0.38$ & $3.17 \pm 0.58$ \\
$\mathrm{t}$ & 0.617 & 0.714 \\
$\mathrm{P}$ & 0.541 & 0.481 \\
\hline
\end{tabular}

3.3 Comparison of the incidence of complications between the two groups

There was no significant difference in the incidence of various complications between the two groups $(\mathrm{P}>0.05)$ (Table 3).

Table 3. Comparison of the incidence of complications between the two groups (n/\%)

\begin{tabular}{ccccc}
\hline Group & Infection & Pleural effusion & Air leakage & Pulmonary infection \\
\hline Study group $(\mathrm{n}=15)$ & $1(6.7)$ & $2(13.3)$ & $1(6.7)$ & $2(13.3)$ \\
Control group $(\mathrm{n}=15)$ & $2(13.3)$ & $3(20.0)$ & $1(6.7)$ & $1(6.7)$ \\
$\chi 2$ & 0.370 & 0.240 & 0.000 & 0.370 \\
$\mathrm{P}$ & 0.542 & 0.624 & 1.000 & 0.542 \\
\hline
\end{tabular}

\section{Discussion}

Lung cancer is a malignant tumor with a high clinical incidence. Early multiple primary lung cancer is a special type of non-small cell lung cancer. Such patients receive timely targeted surgical treatment for a 2-year survival rate of about $40-90 \%$, and a 5 -year survival rate of about It is $35-75 \%$. CT examination is required before surgical resection of early multiple primary lung cancers to accurately identify whether the patient has metastasis and other problems, and to determine the scope of resection on the basis of preserving healthy lung tissue, through segmental resection, lobectomy, wedge resection and other operations The program achieves the therapeutic effect ${ }^{[3]}$.

\subsection{Analysis of the advantages of bilateral thoracoscopic lung resection during the same period}

The main features of thoracoscopic surgery are minor trauma, accurate removal of the lesion tissue, rapid postoperative recovery, and good incision aesthetics. It has been widely used in clinical surgical treatment of various diseases. The clinical application value of simultaneous and staging bilateral thoracoscopic lung resection is still controversial. The clinical application of staged surgery is widely used, and its main drawback is that the interval between two operations is long. During this period, tumor progression can increase the difficulty of treatment. And the treatment cost is expensive, and the complication rate is high $^{[4]}$. Simultaneous bilateral thoracoscopic pneumonectomy is a clinically widely used early treatment plan for multiple primary lung cancers in recent years. It can effectively solve the problems of tumor progression in staging surgery. The use of thoracoscopy to assist in completing the operation during the operation can significantly reduce surgical trauma and improve the safety of surgery. In the early clinical application, bilateral thoracoscopic pneumonectomy mostly adopts lobectomy and wedge resection. With the development of related technologies, anatomical segment resection is increasingly widely used. This surgical method can be based on complete resection of the lesion. The healthy lung tissue is preserved to the greatest extent, the operation has the characteristics of minimal invasiveness, and the safety of bilateral thoracoscopic surgery in the same period is significantly improved.

Early stage patients with multiple primary lung cancer undergoing thoracic surgery often use double-lumen endotracheal intubation combined with intravenous anesthesia, and need to complete the surgical treatment under one-lung ventilation. Some patients need to remove 
local lung tissue or lung lobes during the operation, or even complete the whole operation. Lung resection. By adopting a staged surgical treatment plan, the residual lung tissue cannot meet the basic requirements of one-lung ventilation, resulting in some patients unable to remove residual lung masses through surgical treatment. At the same time, the number of bilateral thoracoscopic lung resections during the same period is less, which can reduce the cost of surgery, reduce the psychological burden of patients, and significantly shorten the overall recovery time. In addition, the staging operation mode has a time window between two operations, during which the body is prone to form a variety of growth factors and inflammatory factors, which in turn leads to tumor progression. There is no time window between two operations in the concurrent operation mode, and the risk of tumor progression is significantly reduced ${ }^{[5]}$.

\subsection{Data analysis of this study}

In this study, patients undergoing bilateral thoracoscopic pneumonectomy during the same period had no history of lung disease, and there was no abnormality in lung function before operation. The time to get out of bed was $(2.84 \pm 0.22)$ $\mathrm{d}$, and the time to extubation was $(3.03 \pm 0.49) \mathrm{d}$, the overall hospitalization time was (7.28 \pm 1.64$)$ days, and there was no significant statistical difference compared with the control group. There was no significant difference in the incidence of various complications between the two groups. It can be considered that the operation mode at the same time had no adverse effect on the postoperative recovery, did not lead to a significant increase in the incidence of complications. There was no significant difference in operation time and intraoperative blood loss between the two groups. The VAS score of the patients in the study group was higher than that of the control group on the first postoperative day, and the total hospital stay and total operation costs were lower than those in the control group. It can be considered that the same operation can shorten the overall treatment Time, reduce the cost of surgery. In order to ensure the smooth completion of bilateral thoracoscopic lung resection during the same period, CT scans must be used to accurately locate the lesion tissue before the operation, and the vital signs of the patient must be closely monitored during the operation to ensure the safety of the operation. At the same time, the results of this study showed that the VAS scores of the patients in the study group were significantly higher than those in the control group on the first day after surgery. It can be considered that bilateral thoracoscopic lung resection in the same period can lead to postoperative pain aggravation. For this reason, postoperative analgesia interventions need to be strengthened, and instruct patients to carry out respiratory function training to prevent lung infections.

\subsection{Precautions related to bilateral thoracoscopic lung resection during the same period}

In order to ensure the efficacy and safety of bilateral thoracoscopic pneumonectomy during the same period, physicians need to strictly grasp the indications of surgery, assess whether the patient has adhesions in the thoracic cavity, whether there is distant metastasis of the lesion, whether there is abnormal cardiopulmonary function, etc., if the patient does not meet the surgical guidelines, other treatment options are required. During the operation, doctors need to perform detailed operations such as anatomy and closely monitor the changes in the patient's vital signs. In order to relieve postoperative pain, promote sputum and cough, they can choose $16 \#$ gastric tube and other thin tubes for bilateral chest drainage. Respiratory management needs to be actively carried out after the operation, and intervention measures such as bedside fiberoptic bronchoscopy can be used to reduce the incidence of lung infections.

Based on the above analysis, it can be seen that the simultaneous bilateral thoracoscopic pneumonectomy for patients with early multiple primary lung cancer has a significant effect and high safety, and can be comprehensively promoted in medical institutions at all levels. At the same time, the total number of patients included in this study is small, there is a lack of comparative research and analysis of the same type of data, the total research time is short, and the process design needs to be continuously improved. The clinical application value of simultaneous bilateral thoracoscopy pneumonectomy in the treatment for patients of early multiple primary lung cancer at the same time still needs continuous research and analysis.

\section{References}

[1] Guo JC, Lu JB, Qiao CR, et al. Application of thoracoscopic paravertebral space tube analgesia in thoracoscopic lobectomy[J]. Chinese Journal of Endoscopy, 2021, 27(2): 8-13.

[2] Li LB, Niu XR, Li D, et al. The efficacy and complications of thoracoscopic lobectomy and conventional thoracotomy in the treatment of lung 
cancer[J]. Practical Journal of Cancer, 2020, 35(1): 91-93,103.

[3] Wang H. Comparison of curative effect between full thoracoscopic lobectomy and traditional open thoracic surgery in the treatment of early stage non-small cell lung cancer[J]. Forum on Primary Medicine, 2020, 24(1): 60-61.

[4] Wang ZZ. Comparison of single-port video-assisted thoracoscopic surgery and thoracotomy for long lobectomy in the treatment of early lung cancer[J]. Contemporary Medicine, 2020, 26(19): 141-142.

[5] Liu HT, Wang LB, Qi WB, et al. Application of three-dimensional reconstruction and virtual surgical planning in thoracoscopic anatomical segmentectomy[J]. Modern Chinese Doctors, 2020, 58(32): 4-7, 193. 